2019-06-14

Management of plaque in people experiencing homelessness using 'peer education': a pilot study

\title{
Paisi, Martha
}

http://hdl.handle.net/10026.1/14662

\subsection{8/s41415-019-0361-0}

British Dental Journal (BDJ)

Springer Nature [academic journals on nature.com]

All content in PEARL is protected by copyright law. Author manuscripts are made available in accordance with publisher policies. Please cite only the published version using the details provided on the item record or document. In the absence of an open licence (e.g. Creative Commons), permissions for further reuse of content should be sought from the publisher or author. 


\section{Management of plaque in people experiencing homelessness using 'Peer Education': A} pilot study

\section{Abstract}

Introduction: People who experience homelessness have poor oral health and limited access to dental services.

Aim: To examine whether 'Peer Education' could yield improved plaque management among people experiencing homelessness.

Methods: A quasi-experimental, one-group pretest-posttest study was conducted, with follow-up at 1 and 2 months. Participants were living in temporary accommodation in Plymouth, UK. Plaque levels were assessed using the Simplified Oral Hygiene Index. A questionnaire and the Oral Health Impact Profile (OHIP-14) were administered. Patient satisfaction and barriers to dental care were explored by interviews.

Results: The baseline sample included 24 people with a mean age of $36.88 \pm 10.26$ years. The mean OHIP-14 score was $25.08 \pm 19.56$; finding it uncomfortable to eat and being embarrassed attracted the highest values $(2.46 \pm 1.53$ and $2.33 \pm 1.63$, respectively). Plaque levels decreased by month 1 and month 2 , though the changes were not statistically significant. Positive changes in confidence in tooth brushing at month 2 were identified $(\mathrm{p}=0.01)$

Conclusion: Experiencing pain and the opportunity to access treatment were key drivers of study participation. The study indicated that it is feasible to conduct oral health promotion projects for people in temporary accommodation. Adequately powered studies examining the impact of peer education on improving homeless people's oral health are warranted. 


\section{Background}

2 Homelessness has risen significantly over the last decade in the UK, where more than

3300,000 people are currently homeless. ${ }^{1}$ A wealth of literature shows that when being

4 homeless it impacts negatively on an individual's health and overall wellbeing., ${ }^{2,3}$

5 Extensive research worldwide has shown a link between homelessness and poor oral

6 health. ${ }^{4,5}$ Several studies in the UK have shown that people experiencing homelessness have

7 higher levels of untreated dental decay and periodontal disease compared to the general

8 population. $^{6,7,8}$ Contributing factors include unhealthy eating habits and oral hygiene

9 practices, challenging living circumstances, mental health and dependency issues (i.e. illicit

10 substances and alcohol), and low use of dental services. ${ }^{6,8,9}$ With these factors in mind, there

11 is a need to establish interventions that improve the ability of people who experience

12 homelessness to care for their oral health and improve access to services. ${ }^{10}$

'Peer Education' is an approach where 'educators', who have personal experience of an issue themselves (in this case, experience of homelessness) work to raise awareness of health issues among vulnerable people and encourage them to change their lifestyle and engage with services. ${ }^{10}$ One methodology often employed is motivational interviewing, a process of 'working with patients that activates their own motivation and resources thereby enabling them to change their behaviours'. ${ }^{11\left(\mathrm{p}^{785)}\right.}$ It has been shown to have great potential in assisting people with poor oral health and established periodontal disease. ${ }^{6,11,12}$

\section{Aim and objectives}

The aim of the study was to examine whether 'Peer Education' could yield improved plaque management among people experiencing homelessness. 


\section{Methodology}

\section{Study design}

3

4

5

6

7

This was a quasi-experimental, one-group pretest-posttest pilot study, entitled 'Teeth Matter'.

It was a collaborative project between the University of Plymouth Faculty of Medicine and

Dentistry (Peninsula Dental School), Peninsula Dental Social Enterprise (PDSE), the homelessness and health charity Groundswell, and a residential centre providing temporary accommodation to homeless people in Plymouth.

\section{Ethical approval}

Approval to conduct the research was obtained from the Faculty of Health and Human Sciences Research Ethics Committee, University of Plymouth (ref: 17/18-854).

\section{Study design development-}

The research team from the University of Plymouth, the peer researcher and educator as well as the Director of Research from Groundswell, and the lead volunteer from the residential homeless centre were involved in developing the study design and materials used in the study. The team all contributed to the development of the protocol, the process of recruiting participants and the content of the study material (e.g. information sheet, consent form, posters etc). With regard to the latter, the lead researcher would develop the first draft and then the rest of the team would comment on the content, design, language, appeal and acceptability of the material.

Two research focus groups with residents in the homeless residential centre (led by the peer researcher) $(\mathrm{N}=11)$, and interviews with relevant stakeholders (i.e. care providers, support workers, dentists, academics and others) $(\mathrm{N}=12)$, were carried out in order to explore optimal ways of engaging with the target population. The findings also formed the basis to inform the 
1 study design and subsequent intervention. All participants provided written informed consent

2 and all the interviews were audio recorded. Refreshments were provided for participants in

3 the focus groups. The findings of the focus groups and the interviews will be the subject of

4 another paper.

\section{$5 \quad$ Recruitment}

A convenience sample was drawn from people living in the residential centre. Participants had to be at least partially dentate (have at least two of the six possible surfaces needed for the calculation of Simplified Oral Hygiene Index $)^{13}$, aged 18 years or over, and based on the opinion of the centre's lead volunteer (in consultation with support staff) able to provide informed consent without their capacity being compromised either by being intoxicated, or being under the influence of drugs or alcohol. Any of the participants who did not meet these criteria at the onset of the study were excluded. Mild intoxication to prevent symptoms of withdrawal was considered acceptable on a case-by-case basis. Those with an acute episode of mental ill health or who were severely intoxicated at the follow stages (in a way that the lead volunteer/support staff believed they were not able to participate in an informed basis), were informed that their absence from a study stage did not preclude them from the next phase.

After obtaining approval from the centre manager, a leaflet/information sheet was distributed to potential participants with the help of the centre's support workers and lead volunteer. The lead researcher and a Groundswell peer researcher (with lived experience of homelessness) visited the centre in February 2018 to present the study in an informal setting, and respond to any questions participants might have had prior to involvement in the study. Baseline, 1- and 2-month follow-up assessments were conducted at the centre in March, April and May 2018, 
1 respectively. Before each visit, the lead volunteer gave a reminder sheet to the study

2 participants confirming the day of the study and time of appointment. During all the study

3 stages and following the clinical assessment, participants were offered refreshments.

4 Participants provided a signed consent form for their participation in the study.

\section{$5 \quad$ Baseline assessments}

\section{Clinical}

7 The clinical examination was conducted in a room at the residential centre used by other

8 healthcare professionals. An experienced primary care dentist, blinded to the study

9 hypothesis and with past experience of providing epidemiological fieldwork for the Adult

Dental Health Survey (ADHS) and the Public Health England's Dental Epidemiology programme, assessed participants' plaque levels using the Simplified Oral Hygiene Index (OHI-S). ${ }^{13}$ The BASCD criteria were used for the visual examination of teeth that were decayed (D), missing (M) or filled (F). ${ }^{14}$ The threshold for recording caries was dentinal involvement $\left(D_{3}\right)$. The degree of urgency for any treatment was divided into 'routine examination', 'routine treatment', 'urgent' and 'fast track'. This was based on the 2009 ADHS participant feedback categories. ${ }^{15}$ Fast track and urgent referrals were discussed sensitively with participants and made immediately after the initial assessment.

\section{Sociodemographic characteristics and oral health-related quality of life}

Information on participants' demographic and lifestyle characteristics, oral health and dietary habits was collected using a questionnaire developed specifically for the project. This was previously pilot tested in a Plymouth forum accessed by homeless people $(\mathrm{N}=10)$. The Oral Health Impact Profile (OHIP-14) ${ }^{16}$ was used to assess oral health-related quality of life. Both the questionnaire and OHIP-14 were administered by study personnel. 
2 Following the clinical examination, a dentist demonstrated tooth brushing to each participant.

3 A Groundswell peer educator then used motivational interviewing to develop the

4 participants' sense of self-efficacy regarding oral health. The peer educator provided basic

5 oral health information based on Delivering Better Oral Health guidelines, ${ }^{17}$ focusing

6 primarily on brushing twice daily for two minutes with fluoride toothpaste, and reducing the

7 amount and frequency of sugary food and drinks. The importance of oral health to the overall

8 wellbeing as well as the importance of maintaining preventive oral care activities after dental

9 treatment, were highlighted. The peer educator had received a 2-day motivational

10 interviewing course by accredited Groundswell trainers ${ }^{18}$ and basic training by Plymouth

11 researchers on the 'Delivering better oral health guidelines' ${ }^{17}$ that were used as part of the

12 intervention.

13 'Goody bags' containing toothpaste, a toothbrush, a timer and a leaflet designed specifically

14 for the study and which included basic oral health messages, were given to all participants. A

15 poster with the same messages included in the leaflet was placed in each communal bathroom

16 at the centre. The resources developed as part of this project will be provided in another

17 publication.

\section{Follow-up}

At one and two months after the intervention, the OHIS-S, OHIP-14 assessments, and the questionnaire were carried out. New toothpastes were also provided and another poster (with more visuals and less words than the first one) promoting the message to brush teeth twice a day for two minutes was placed in all communal bathrooms. 


\section{Process evaluation}

2 At the end of the study, semi-structured interviews were conducted with all participants

$3 \quad(\mathrm{~N}=15)$ to assess their experience of, and satisfaction with, the intervention. The barriers and

4 enablers to brushing their teeth were also explored. All the interviews were audio-recorded

5 and transcribed verbatim. The question framework for the interview is available upon request.

6 Upon completion of the study, participants were given certificates of appreciation and thank

7 you cards. They were also given the opportunity to access free dental treatment provided by

8 Peninsula Dental Social Enterprise (PDSE).

\section{Analysis}

Bivariate analysis was used to examine the association between age and OHIP-14 scores, and age and DMFT, at baseline. The Wilcoxon signed ranked test was used to examine change in plaque levels (OHI-S) and participants' confidence in using the correct tooth brushing technique after 1 and 2 months. SPSS (v21) was used for all statistical analyses. Statistical significance was indicated by a $\mathrm{p}$ value of less than $5 \%$. The transcripts of the semi-structured interviews that conducted at the end of the study (i.e. process evaluation) were managed using NVivo software (v11). The themes were deductively driven to focus the analysis on areas of interest for the intervention evaluation and ongoing development, The Strengthening the Reporting of Observational Studies in Epidemiology (STROBE) guidelines were used to report the study.

\section{Results}

Figure 1 presents the number of participants at each stage and provides reasons for drop-outs. 19 and 15 people completed the 1 and 2 month follow-up, respectively. 
2 The sample at baseline included 24 British men aged on average 36.88 (SD \pm 10.26, range:

3 21.20-58.60) years. Supplementary File 1 presents their sociodemographic characteristics.

4 The response rate for the questionnaires at each study stage was $100 \%$. The majority of 5 participants were single $(\mathrm{N}=19)$. Most participants $(91.7 \%, \mathrm{~N}=22)$ were smokers, whilst $6 \quad 66.7 \%(\mathrm{n}=16)$ reported that they did not drink alcohol most days. Seventeen $(70.8 \%)$ stated 7 that they had used recreational drugs, and seven were currently using drugs. Eight people (33.3\%) had been prescribed methadone in the past with two of them currently being prescribed it. Amongst those prescribed methadone, four reported that it was sugar-free and the rest that it was sugar-containing. Approximately half of the sample $(54.2 \%, \mathrm{~N}=13)$ reported that they currently experienced physical health problems, and $62.5 \% \quad(\mathrm{~N}=15)$ reported suffering mental health issues. Almost all were registered with a GP $(95.8 \%, \mathrm{~N}=23)$, but none were in ongoing care with a dentist and only three had visited a dentist in the last year. The last time that most participants had visited a dentist was because they had toothache or some other problem $(\mathrm{N}=19,79.2 \%)$; four had attended for routine checkups $(16.7 \%)$ and one because the dentist had sent them a reminder (4.2\%).

Nearly $63 \%$ of the sample $(\mathrm{N}=15)$ reported at baseline that they brushed their teeth less than twice a day, and $37.5 \%(\mathrm{~N}=9)$ reported that they were brushing their teeth twice or more a day. At the 1-month follow up, the corresponding percentages were $47.4 \%(\mathrm{~N}=9)$ and $52.6 \%$ $(\mathrm{N}=10)$, respectively. At the 2-month follow up, these figures were $28.6 \%(\mathrm{~N}=4)$ and $71.4 \%$ $(\mathrm{N}=10)$. The proportions at 1 and 2 -month follow-up were not statistically significant compared to the baseline ones $\left(\mathrm{p}_{1}=0.625\right.$ and $\left.\mathrm{p}_{2}=0.250\right)$. Eight $(33.3 \%)$ of the participants at baseline had sugary food items or drinks four times and more daily. Approximately half of the sample had their sugary items or drinks in between meals $(54.2 \%, \mathrm{~N}=13)$. 
2 Table 1 shows the clinical characteristics of the study sample. All participants had experience 3 of decay (DMFT>0). The mean DMFT of the sample was 16.58 ( $\mathrm{SD} \pm 8.07)$, the mean DT 4 was $6.75(\mathrm{SD} \pm 5.76)$, the mean $\mathrm{MT}$ was $7.75(\mathrm{SD} \pm 6.78)$, and the mean $\mathrm{FT}$ was 2.08 5 ( $\mathrm{SD} \pm 2.93)$. One participant had a partial upper denture. With regard to treatment needs, one 6 participant was referred for a fast-track examination due to a suspicious oral mucosal lesion 7 and five for urgent care due to pain. Upon study completion all participants but two were assessed as needing routine treatment.

The frequency of problems reported by participants related to oral conditions in the preceding 12 months is presented in Table 2. The mean values of items are presented below (Table 3).

\section{Please insert Table 2 here}

Please insert Table 3 here

The total OHIP-14 score was $25.08 \pm 19.56$. The highest values were obtained from the items: finding it uncomfortable to eat $(2.46 \pm 1.53)$, being embarrassed $(2.33 \pm 1.63)$ and being selfconscious $(2.21 \pm 1.56)$.

At baseline, there was a statistically significant positive relationship between OHIP-14 total score and age (Spearman's rho $=0.631, \mathrm{p}=0.001)$. A significant moderate relationship was also found between age and DMFT (Spearman's rho=0.667, $\mathrm{p}<0.001$ ). However, age and OHI-S at baseline did not relate significantly (Spearman's rho=0.131, $\mathrm{p}=0.540$ ). Furthermore, DMFT, but not OHI-S, related to OHIP-14 (rho=504, $\mathrm{P}=0.012$; rho= $-0.118, \mathrm{P}=0.582$ ).

The OHI-S decreased from baseline (median=0.60) to month $1($ median $=0.40)$ and to month 2 follow-up (median= 0.35$)$. However, the changes were not statistically significant $(p=0.82$ 
1 and $\mathrm{p}=0.23$, respectively). The confidence of participants in using the correct tooth brushing

2 technique significantly improved at month $2(\mathrm{~N}=15, \mathrm{Z}=-2.53, \mathrm{p}=0.01)$.

\section{$3 \quad$ Process evaluation interview results}

\section{$4 \quad$ Key drivers for study participation}

5 The main motivator for participants taking part in the study was the opportunity to access 6 dental treatment in order to improve the condition of their teeth. Feelings of embarrassment 7 and functional limitation, including pain and difficulty in eating, were common reasons

8 reported by participants for wanting dental treatment. Participants reported that although 9 being very conscious of the condition of their mouth, it was very hard to access services. Some highlighted the impact that dental treatment would have on their confidence and selfesteem as they felt being judged because of the appearance of their teeth.

"The society is now used to bright white smiles and that aint it? I have been called... all sort of things" (Participant 4)

"I know for fact that I have got something to gain for... I have been like this now for 3 years now. And it is very embarrassing when I go outside and talk to people. 'Cause I am very conscious that they notice I have no teeth. And I don't want to be like that.... And I want to be able to talk to someone with smile, teeth, everything ..." (Participant 1)

Participants also acknowledged that seeking care was part of seeking a new start in their lives, particularly in relation to job seeking.

"If you want to move on and get job, you know, move on to flats and stuff, you have to have good teeth really aint you" (Participant 7)

"I don't want to go back to fishing anymore. I have done it for twenty years. I have one son, one life and that all what I want to do now. I want to get in touch with other jobs than fishing.....that's what I am working one at the minute...Just be happy and with a nice white smile. It will make a big difference. .." (Participant 6) 
1 The participants were very satisfied with the project and there was nothing negative that they

2 reported about the study. What they liked most was the opportunity to get treatment after the study, the friendliness and professionalism of the staff, the fact that barriers between them and dentists had broken down and that they had been shown how to brush their teeth. For some, emphasis on the personal qualities of the study team related to previous bad experiences when visiting a dentist. Furthermore, some of the participants acknowledged that prior to the study the only time that they were shown how to brush teeth was when they were at school. Being aware that the advice to patients can change, they highlighted that they were not confident (prior to the study) that they were using the correct tooth brushing technique. Little waiting time in between study stations was also greatly valued by the participants.

'It's a really nice study, to be honest with you. Everybody is really polite and nice. And you don't feel you are in danger in any way, shape or form. I mean, I can remember being a kid and going to the dentist. It was the scariest thing you ever come across.' (Participant 9)

\section{Peer research and education}

Although participants acknowledged that when it came to dental treatment, they would "obviously want a trained professional", the presence and support from someone with lived experience of homelessness made them feel more connected with the project. It also made it easier for them to engage in a discussion. This is because they had been through the same 'journey' and could speak in a language they understood ("my kind of language"). Knowing the background of the peer educator also made it easier for them to take her advice. The fact that she did not over-explain was also highlighted as one of the elements of peer education that they liked.

"Because if they've been through it, then they can understand it and properly input it to me, in a better way that someone who hasn't lived it." (Participant 5)

"I understood what they had experienced, respected them and felt it back-two-way respect. They were good as gold. They are good people who we need involved in helping us" (Participant 2) 


\section{Barriers to brushing teeth during the study}

3 Established unhealthy habits and mental health issues were common barriers to brushing

4 teeth during the study. The latter also hampered participants' ability to participate in other 5 activities unrelated to dental health. Some also reported that tooth sensitivity made it difficult 6 for them to brush their teeth.

'It's more habit than anything' (Participant 1)

..at least three or four times a week I get really bad depression and I don't really want to participate or do anything really... I sometimes don't even get dressed, I don't bother with my meals ...I just stay in my room and I don't get out.' (Participant 3)

\section{Enablers to brushing teeth during the study}

In terms of enablers, provision of information (i.e. leaflet) and resources (i.e. toothbrush, timer) were among the most common reasons participants gave as to what helped them brush their teeth during the study. Furthermore, realising that brushing was effective, had helped remove bad breath and that it would prevent further deterioration of the teeth (which would have eventually led to embarrassment), was another reason that motivated participants to brush their teeth. For some, this related to emotional attachment to their children.

"I don't want my daughter to see me with horrible teeth. I pick her up from the school. I don't want her to see her dad with no teeth or bad teeth, I don't want that." (Participant 4)

\section{Discussion}

The current study is the first in the UK to carry out a dental intervention for people who are homeless using the concept of peer education. It demonstrated that people who experience homelessness have a high number of missing and untreated decayed teeth, as well as a low number of filled teeth. Plaque levels were generally low. These results are consistent with previous findings. ${ }^{6,19}$ A low F component of the DMFT indicates poor access to dental care, 
1 and is confirmed by the finding that none of the participants were registered with a dentist. ${ }^{7}$

2 Treatment needs were also extensive with the majority of participants requiring more than a routine examination. This suggests that more accessible dental care pathways are needed for people experiencing homelessness in Plymouth. Peninsula Dental Social Enterprise is currently responding by developing a service where people who are homeless can access dental treatment free of charge.

This study showed that reported tooth bushing frequency at baseline was low, whilst tobacco use and sugar intake between meals were high. Taking into account that low tooth brushing frequency, tobacco use and snacking in between meals are associated with poor oral health, ${ }^{17}$ future studies to identify successful means to promote preventive daily oral care activities and improve self-confidence and efficacy for people who are homeless are needed. Exploring the reasons for poor adherence to preventive activities can help the focusing of the messages to promote behavior change. ${ }^{20}$ Consideration should also be given to the fact that people in temporary accommodation may not have a lot of options with regard to the food choices available. This indicates the need for a collaborative approach involving management, as part of creating a supportive environment to behavior change. ${ }^{20}$ Furthermore, when clinically appropriate, doctors should be encouraged to prescribe sugar-free methadone for those who are recovering from drug addiction.

Following the intervention, plaque levels improved, however the changes were not statistically significant. Given the extensive treatment needs and poor oral health among participants and the anticipated small effect size, it is encouraging to note the positive, albeit non-significant reduction in plaque levels along with clear improvement in toothbrushing confidence. It demonstrates that peer education along with demonstration of toothbrushing technique by a qualified professional could be useful in reducing plaque for people who are homeless and promoting their engagement with dental care. Further, adequately powered 
1 studies including randomised controlled trials are needed to examine the effectiveness of this

2 concept in improving oral health for people experiencing homelessness.

3 Embarrassment and self-consciousness were key issues affecting participants' oral health-

4 related quality of life. Previous research has indicated that embarrassment at the condition of

5 their teeth and the subsequent impact on self-esteem can itself act as a barrier preventing

6 homeless people from visiting a dentist. ${ }^{6,8,9}$ The condition of the mouth can also make

7 homeless people self-conscious when interacting with others and it also affects their

8 confidence when seeking to enter the job market. ${ }^{6}$ In the present study, embarrassment

9 appeared to be a significant factor motivating participants to seek treatment. Participants also acknowledged that seeking dental care felt like a step towards reclaiming their life. This finding, in line with previous studies, gives support to the hypothesis that oral health intervention can become part of a homeless person's journey towards stability and potentially improve their quality of life. ${ }^{6,21}$ Giving participants in research projects the opportunity to access free dental treatment is highly recommended and could also act as an incentive for both participation and retention in the study.

The intervention, which was evidently well received by the participants, took into account the understandable embarrassment and inhibitions of people who have a history of marginalisation and difficulties in accessing services. The absence of any negative comments for the study was very encouraging but may also indicate low expectations of people who experience homelessness for efforts aiming to improve their oral health and their appreciation for projects aiming to improve their health. Engaging with those who support homeless people and giving people who have experienced homelessness the opportunity to participate in the development of the project has proven invaluable in ensuring participants' enjoyment of and commitment to the study. The significance of input of both peer researchers and key workers in the study design, development of research tools, tailoring the health messages to 
1 the particular group, and in study implementation cannot be overstated. Tailoring messages

2 can also help maximise behavior change. ${ }^{20}$

3 Peer researchers and educators are experts by experience and thus their input into a project is essential in ensuring its acceptability to the participants. Regardless of an in-depth knowledge of the available literature in the field, there were crucial to this study elements that the researchers and clinicians were unable to confidently decide upon until input was provided by

7 Groundswell (i.e. reference to drug addiction, ill mental health, dress code and others).

8 Furthermore, the semi-structured interviews evidenced that the presence of someone who has

9 shared their experience made participants feel that they could connect and be better understood. Prior to the conduct of any study adopting the concept of peer education, it would be valuable to explore with patients/participants what they would be looking for in a 'peer'. For example, if it might go further than being someone who has also experienced homelessness, to be someone they can identify with from a wider lifestyle context, e.g. from the same local community as themselves, or a similar small city, same gender, similar route into homelessness. It could also be someone who lives nearby and knows the local challenges homeless people experience and the places they tend to frequent, and services they use.

The link with the lead volunteer within the centre was also pivotal for the recruitment, follow-up and management of participants. This also helped established rapport between the research team and the participants, and ensure that the intervention was acceptable to the particular group of participants. Actively involving the staff in project development and management, and raising their awareness and knowledge on oral health matters (based on evidence based knowledge) can help empower and support behavior change and ensure the sustainability of the project. ${ }^{20}$ Loss of participants at follow-up was due to circumstances beyond their control and when developing projects with follow up stages, consideration should be given to the changing living circumstances of people who live in temporary 
1 accommodation. Although a longer-term study would be better able to assess the impact of

2 behavioural change, this may also result in higher attrition rates due to the aforementioned

3 reason.

\section{$4 \quad$ Limitations}

5 The present study is based on a small convenience sample of people who are homeless and it 6 was not powered to detect statistically significant changes in plaque measurements. However, 7 it identified positive trends in plaque reduction and provides estimates of the variability of the main outcome to inform power calculations for a subsequent full-scale randomised control

9 trials. It also provides estimates for the calculation of the likely response and attrition rates.

10 Furthermore, although generalisability may be limited, our participant characteristics reflect national figures, such as high number of males and British nationals, high prevalence of mental health problems and poor oral health habits.

Periodontal conditions are common among people experiencing homelessness and, therefore, the use of a periodontal index such as Basic Periodontal Examination (BPE) ${ }^{22}$ would have provided a better picture of the treatment needs of our participants. Although we initially planned to include the BPE, it was later decided that it would be better to omit this index as it may have caused discomfort to participants and could have potentially worsened their anxiety.

\section{Conclusions}

Poor oral health is common among people who experience homelessness. The present study indicated that it is feasible to conduct oral health promotion projects for people in temporary accommodation. The need to manage pain and to have access to dental care were identified as the two major drivers to participation in the project. 
1 Peer education could be a useful means of improving plaque management of people who are

2 homeless and of promoting their engagement with dental care. Well-powered studies

3 examining the effectiveness of this concept on improving self-care, oral health and access to

4 dental services are warranted.

\section{$5 \quad$ Key points}

- The first project in the UK to explore the concept of Peer Education in improving plaque management among people who are homeless;

- Provides an insight into engaging people experiencing homelessness with dental care;

- Identifies the main issues affecting homeless people's oral health-related quality of life.

\section{Acknowledgements}

The authors are extremely thankful to the Oral and Dental Research Trust for providing funding for this study. We also thank the charity Well Connected for providing the goody bags distributed at baseline, and PDSE for providing us with the clinical equipment and consumables. Many thanks to Stephan Morrison from Groundswell for leading the focus groups. A very big thank you is due to the dental team and administration staff at PDSE, particularly Christina Worle, for providing care to our participants free of charge. The study would not have been possible without the support of the lead volunteer and our participants.

\section{Conflict of interest}

The authors declare no conflicts of interest. The funders had no role in the analysis or interpretation of data. 


\section{References}

1. Shelter. Far from alone. Homelessness in Britain in 2017. London: Shelter, 2017.

2. Healthy London Partnership. Health care \& people who are homeless. Commissioning Guidance for London. London: Healthy London Partnership, 2016.

3. Thomas B. Homelessness kills: An analysis of the mortality of homeless people in early twenty-first century England. Sheffield: Crisis, 2012.

4. De Palma P, Frithiof L, Persson L, Klinge B, Halldin J, Beijer U. Oral health of homeless adults in Stockholm, Sweden. Acta Odontol Scand. 2005; 63(1): 50-5.

5. Figueiredo RL, Hwang SW, Quiñonez C. Dental health of homeless adults in Toronto, Canada. J Public Health Dent. 2013;73(1):74-8.

6. Coles E, Edwards M, Elliot GM, Freeman R, Heffernan A, Moore, A. The Oral Health of Homeless People across Scotland: Report of the Homeless Oral Health Survey in Scotland, 2008-2009. Dundee: University of Dundee, Dental Health Services Research Unit, 2011.

7. Daly B, Newton T, Batchelor P, Jones K. Oral health care needs and oral healthrelated quality of life (OHIP-14) in homeless people. Community Dent Oral Epidemiol. 2010; 38(2): 136-44.

8. Hill KB, Rimington D. Investigation of the oral health needs for homeless people in specialist units in London, Cardiff, Glasgow and Birmingham. Prim Health Care Res Dev. 2011; 12: 135-144.

9. Caton S, Greenhalgh F, Goodacre L. Evaluation of a community dental service for homeless and 'hard to reach' people. Br Dent J. 2016; 220(2): 67-70.

10. Groundswell. Healthy Mouths: A peer-led health audit on the oral health of people experiencing homelessness. London: Groundswell, 2017.

11. Kay EJ, Vascott D, Hocking A, Nield H. Motivational interviewing in general dental practice: A review of the evidence. Br Dent J. 2016; 221: 785-791. 
12. Duane B. Psychological approaches to behaviour for improving plaque control. Evid Based Dent. 2017; 18: 3-4. doi:10.1038/sj.ebd.6401213

13. Greene JG, Vermillion JR. The Simplified Oral Hygiene Index. J Am Dent Assoc. $1964 ; 68(1): 7-13$

14. Public Health England. Oral health survey of adults attending general dental practices 2017/18. National protocol. London: Public Health England, 2017.

15. O’ Sullivan I, Lader D, Beavan-Seymour C, Chenery V, Fuller E, Sadler K. Foundation Report: Adult Dental Health Survey 2009 (Technical information). London: The Health and Social Care Information Centre., 2011.

16. Slade GD (1997). Derivation and validation of a short-term oral health impact profile. Community Dent Oral Epidemiolo. 1997; 25: 284-90.

17. Public Health England. Delivering better oral health: an evidence-based toolkit for prevention. Third Edition. London: Public Health England and Department of Health, 2017.

18. William MR, Rollnick S. Motivational Interviewing. Helping People Change. $3^{\text {rd }}$ edition. New York: The Guilford Press, 2012.

19. Waplington J, Morris J, Bradnock G. The dental needs, demands and attitudes of a group of homeless people with mental health problems. Community Dent Health. 2000; 17(3): 134-137.

20. Tahira Akbar T, Turner S, Themessl-Huber, M, Freeman R The Evaluation of HMP Shotts'Oral Health Improvement Project. Dundee: Oral Health and Health Research, Programme, Dental Health Services Research Unit, University of Dundee, 2012.

21. Beaton L, Coles A, Rodriguez A, Freeman R. HoPSCOTCH Report. Homeless People in SCOTland: a process evaluation of a Community-based oral Health intervention - 

the findings of a pilot study from 4 NHS Boards. Dundee: University of Dundee, 2015.

22. The British Society of Periodontology. Basic Periodontal Examination (BPE). Selby: The British Society of Periodontology, 2016.

\section{(1)}

